\title{
OPEN 2D materials coated on etched optical fibers as humidity sensor
}

\author{
Erfan Owji, Hossein Mokhtari ${ }^{\bowtie}$, Fatemeh Ostovari, Behnam Darazereshki \& \\ Nazanin Shakiba
}

In this investigation, etched-fibers are coated by 2D layers such as Molybdenum disulfide $\left(\mathrm{MoS}_{2}\right)$, Molybdenum diselenide ( $\mathrm{MoSe}_{2}$ ) and composition of graphene and graphene oxide (G/GO) to modify humidity sensing. The relative differentiation of attenuations (RDA) in presence of relative humidity $(\mathrm{RH})$ is measured by Optical Loss Test Set at two standard-wavelengths-telecommunication (1310 nm and $1550 \mathrm{~nm}$ ). Results show that the etched single-mode fiber (ESMF) coated with G/GO has relatively high and one by one function for RDA versus $\mathrm{RH}$ (more than $30 \%$ ). Also, its sensitivity and variance are reasonable. The $\mathrm{MoSe}_{2}$ based sensor is applicable at humidity below $30 \%$ because of higher RDA. However, it is not useful at humidity more than $30 \%$ due to the absence of one by one function for RDA versus RH. Besides, ESMF coated with $\mathrm{MoS}_{2}$ has indistinctive behavior and is not useful as a humidity sensor.

Sensors based on optical fibers have a key role in industrial, biomedical, and home security purposes. The great advantages of optical sensors such as high speed, insensitive to electromagnetic interference, anti-explosion, small size, low weight, durable and chemically inert, and low transmission make them practical and affordable ${ }^{1,2}$. However, optical fiber sensors can be used to measuring different physical properties, such as measurements of RI, strain ${ }^{3}$, temperature ${ }^{4,5}$, and humidity ${ }^{4-10}$. The mechanism of these sensors is very dependent on changes in the refractive index ${ }^{3,4}$. Usually, these sensors are made by modifying the geometry of optical fiber such as sidepolished fiber, a long period fiber grating (LPG), and hollow-core fiber sensors that are very complicated ${ }^{5-13}$. Recently, in order to improve the sensing properties, coating of optical fibers by nanomaterials has been used.

The humidity sensor is one of the most widely used sensors. So far, extensive researches have been done on the optical humidity sensors due to their advantages. However, the very low permeability of the protective layer and clad of fiber to the vapor leads to slight changes in the refractive index and so very poor sensitivity and non-linearity response. Researchers used physical and chemical changes on the external surface of optical fiber to improve humidity sensing. Especially, coating of two dimensional (2D) materials have attracted great attention in high-sensitive sensors due to their high surface to volume ratio and other intrinsic properties ${ }^{14-16}$. In addition, the ability to absorb water molecules depends on the surface's functional groups of 2D materials. Electrical gap and consequently refractive index changes with bonding between the water molecule and surface of coated optical fiber.

Graphene $(G)$, as the first experimental 2D materials, has attracted a wide range of interest due to its fascinating electronic, mechanical, and thermal properties such as extremely high carrier mobility, mechanical flexibility, optical transparency, and chemical stability ${ }^{17-23}$. Among other applications, the exceptional surface to volume ratio and high electron mobility in room temperature entitles $\mathrm{G}$ as a promising candidate for sensing applications $s^{24}$. Usually, in the synthesis process, different functional groups create on the surface of $\mathrm{G}$ and change its properties. Oxygen functional groups such as hydroxyl, carboxyl, epoxide, and carbonyl are the main functional groups that convert graphene to graphene oxide (GO). Presence of these groups increases surface and interlayer adsorption of water molecules through the polar bonding ${ }^{11,16,25}$.

Different humidity sensors based on optical fiber with different geometrical shape coated with G or GO have been investigated. Although some groups have used humidity sensors based on fiber optic with reduced graphene coating due to changes in carrier density by variations of relative humidity ${ }^{10,12,16,26,27}$, others have preferred graphene oxide because of its hydrophilic property and changes in its refractive index by adsorption of water molecules between its layers ${ }^{11,28-30}$. It seems that by use of $\mathrm{G}$ and $\mathrm{GO}$ simultaneously, make it possible to use the benefit of both them in humidity sensing based on optical fiber which has not been studied until now.

Also, the layered transition metal dichalcogenides (TMD) materials-a new family of $2 \mathrm{D}$ materials have attracted huge attention due to tunable direct band gap, high mobility, low-level toxicity, and large surface area ${ }^{15,31,32}$. The monolayer of Molybdenum Disulfide $\left(\mathrm{MoS}_{2}\right)$ and Molybdenum Diselenide $\left(\mathrm{MoSe}_{2}\right)$ is an outstanding example, in which molybdenum atoms are sandwiched between two layers of Sulfur and Selenium 

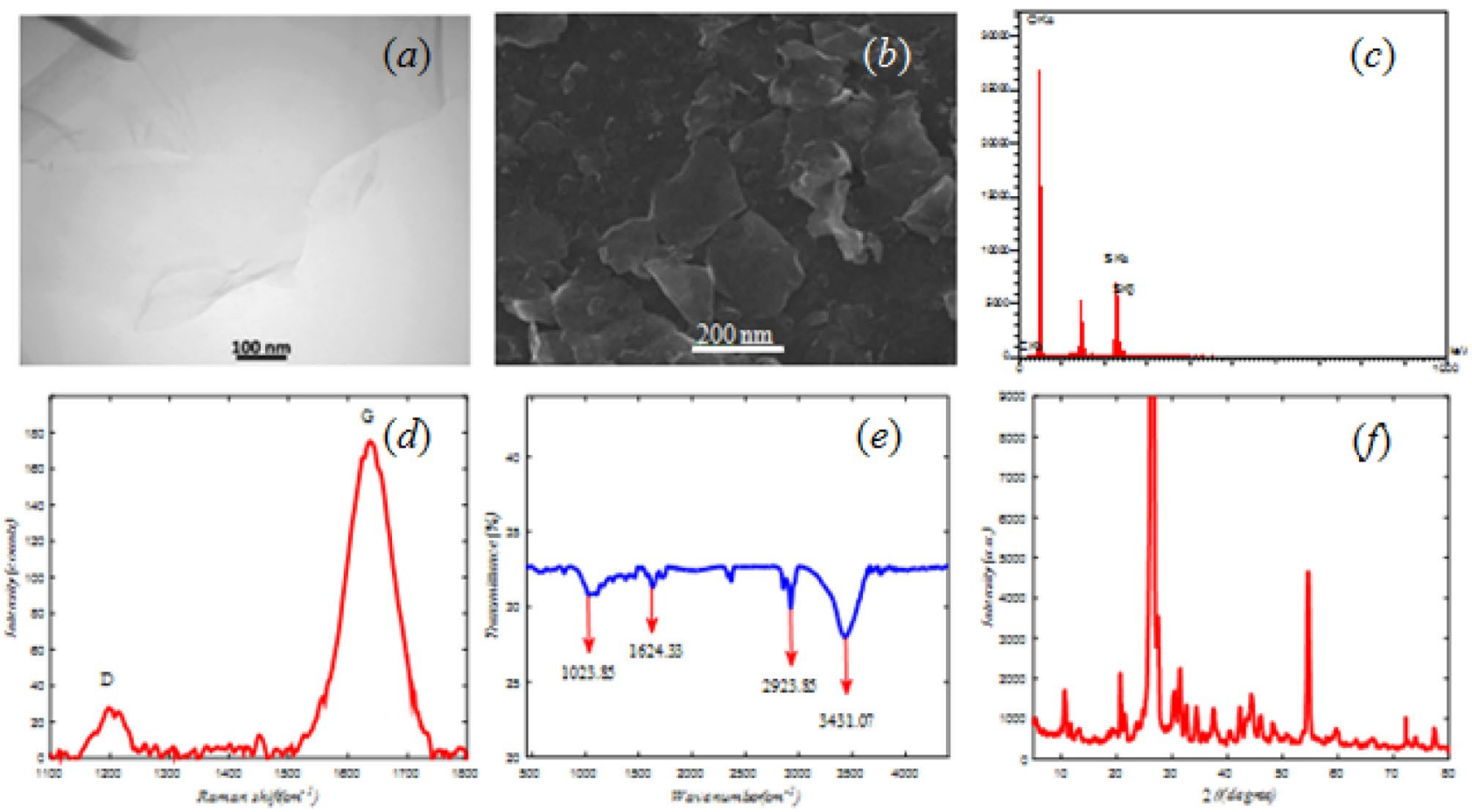

Figure 1. (a) TEM, (b) SEM, (c) EDX, (d) Raman, (e) FTIR, and (f) XRD analysis of G/GO.

atoms respectively. The humidity sensor based on the side-polished optical fiber coated with these nanosheets have been investigated at $1550 \mathrm{~nm}$ wavelength ${ }^{33-35}$. The use of mechanical polishing method results in insertion loss of the side-polished fiber and smoothness of edges is difficult.

However, chemical polishing methods have preserved the cylindrical shape of the optical fiber and is much simpler and more practical than other methods. As far as we have researched, so far the optical fibers that have been chemically thinned and layered with 2D materials have not been applied as humidity sensor.

In this study, for the first time, chemical etching the surface of single-mode optical fiber (SMF) by hydrofluoric acid (HF) as a simple polish method and coating 2D materials such as the composition of G and GO (G/ $\mathrm{GO}$ ) to use the advantage of both them, $\mathrm{MoS}_{2}$ and $\mathrm{MoSe}_{2}$ on the etched-SMF (ESMF) are used to investigate the humidity sensing properties of optical fiber. So, at first G/GO, MoS 2 and $\mathrm{MoSe}_{2}$ are synthesized and qualified by Scanning Electron microscopy (SEM), Transmission Electron Microscopy (TEM), Energy-Dispersive X-ray spectroscopy (EDX), Raman, FTIR (Fourier-transform infrared spectroscopy) and XRD (X-ray Diffraction) analysis. Then, etched-SMFs are coated by synthesized 2D materials. In the end, the relative differentiation of attenuation (RDA), repeatability, sensitivity, and variance versus relative humidity (RH) at two standard- wavelengths- telecommunication (1310 $\mathrm{nm}$ and $1550 \mathrm{~nm}$ ) are measured.

Also, the behavior of the introduced humidity sensor has been analyzed based on the surface functional groups and the semiconductor type (n-type or p-type) of the coated 2D materials on the ESMF. We have not found this type of analysis in the field of humidity sensing by fiber optic as far as we have studied.

\section{Materials and methods}

In this research, SMF is used as the main part of the optical-humidity sensor. To modify the sensing, the diameter of SMF reduced by corroding it in HF acid and layered with $2 \mathrm{D}$ materials (G/GO, $\mathrm{MoSe}_{2}$, and $\left.\mathrm{MoS}_{2}\right)$ to achieve desirable evanescence field. The Optical Loss Test Set (OLTS) as an analyzer (Optical power source (OPS) and Optical power meter (OPM)) was used to measure RDA versus $\mathrm{RH}$.

Synthesis. Graphene. The G/GO layer is synthesized by Liquid Phase Exfoliation of Graphite Oxide. Usually, the composition of G and GO produces in this method. After synthesis of Graphite Oxide powder by Hummer's method ${ }^{36,37}, 5 \mathrm{mg}$ of it disperses in $10 \mathrm{cc}$ distilled water and sonicates to separate individual Graphite Oxide layers. The transparent liquid above sediments that contain few/mono-layer of G/GO is achieved after centrifugation at $6000 \mathrm{rpm}$ for $3 \mathrm{~h}$. The quality of G/GO layers is investigated by SEM, TEM, EDX, Raman, FTIR and XRD analysis.

The SEM and TEM images display the microstructure of synthesized G/GO (Fig. 1a,b). The corresponding EDX analysis (Fig. 1c) indicates the presence of some impurity in the results and high $\mathrm{O} / \mathrm{C}$ ratio.

The Raman spectra of G/GO layer show two important peaks called D and G bands (D 1197 and $\mathrm{G} \sim 1634 \mathrm{~cm}^{-1}$ ). The $\mathrm{G}$ and $\mathrm{D}$ bands correspond to the presence of $\mathrm{sp}^{2}$ carbon-type structures within the sample, and defects in the hexagonal structure of graphite respectively (Fig. 1d) ${ }^{38,39}$. 

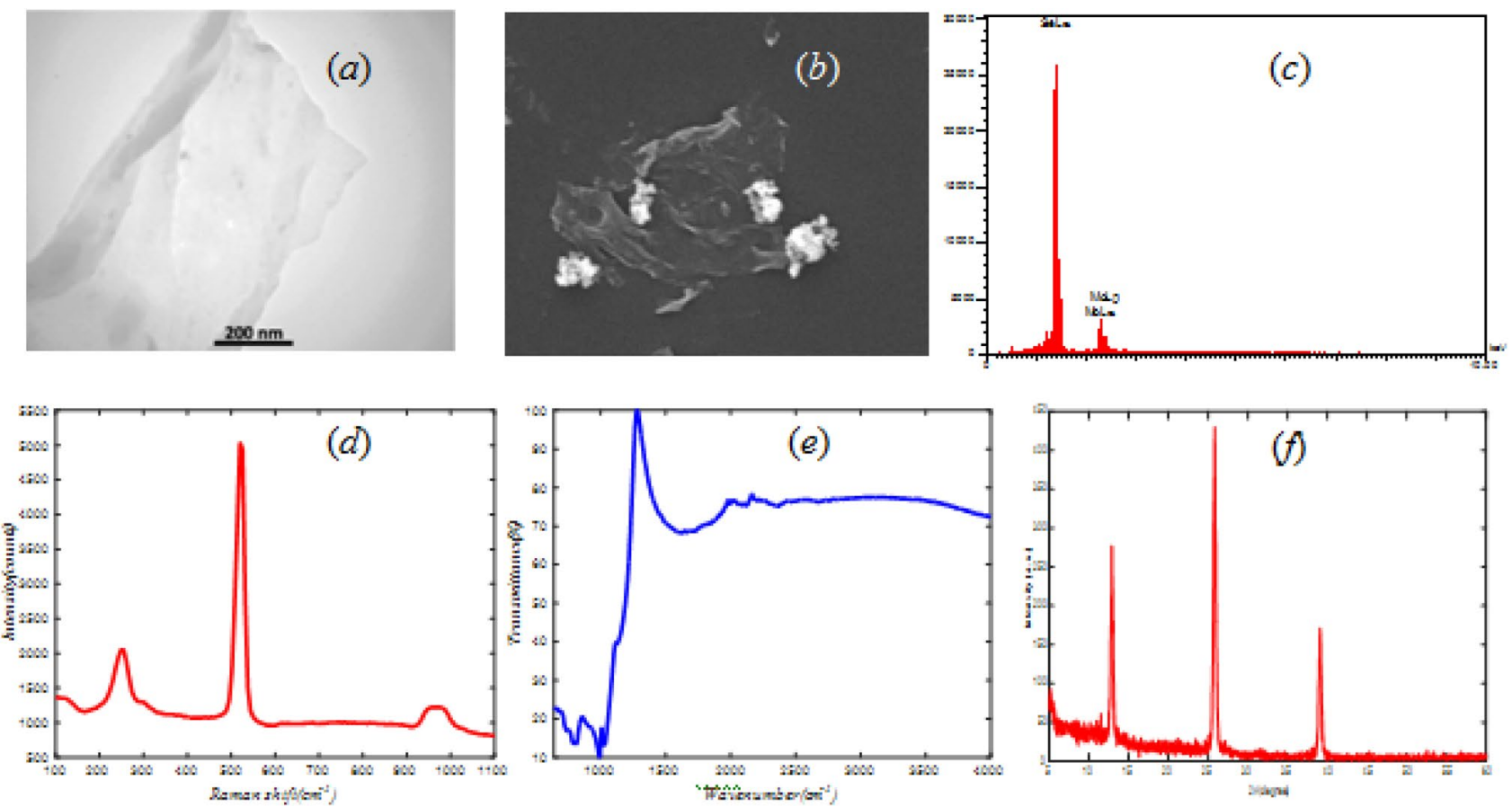

Figure 2. (a) TEM, (b) SEM, (c) EDX, (d) Raman, (e) FTIR, and (f) XRD analysis of MoSe ${ }_{2}$.

The presence of $\mathrm{C}-\mathrm{O}, \mathrm{C}=\mathrm{O}$ and $\mathrm{C}-\mathrm{H}$ bands at 1020,1624 and $2,923 \mathrm{~cm}^{-1}$ respectively are evident from the FTIR spectra (Fig. 1e.). Also, the spread peak around $3430 \mathrm{~cm}^{-1}$ is due to the tensile vibration of phenol $(\mathrm{C}-\mathrm{OH})$ groups $^{40,41}$.

The XRD of G/GO sheets (Fig. 1f) illustrates the peaks at $2 \theta=10^{\circ}(\mathrm{d} 001=0.83 \mathrm{~nm}), 2 \theta=20^{\circ}(\mathrm{d} 002=0$ $0.48 \mathrm{~nm}), 2 \theta=26^{\circ}(\mathrm{d} 002=034 \mathrm{~nm})$ and $2 \theta=55^{\circ}(\mathrm{d} 004=00.17 \mathrm{~nm})$. The peaks at $2 \theta=26^{\circ}, 55^{\circ}$ is characteristic of graphite and after chemical oxidation of it, the inter planar distance increase and the other peaks appear ${ }^{39,42}$.

$\mathrm{MoSe}_{2}$. The synthesis of $\mathrm{MoSe}_{2}$ was down by Solvothermal method ${ }^{24}$ in normal ambient environmental conditions. In this method Se powder, $\mathrm{Na}_{2} \mathrm{MoO}_{4}$, distilled water, and Hydrazine Hydrate are mixed in a container. The materials are stirred constantly and heated with rate $2{ }^{\circ} \mathrm{C} / \mathrm{min}$ for $45 \mathrm{~min}$. Then, the temperature of the homogenize solution is maintained at $120{ }^{\circ} \mathrm{C}$ for an hour. Afterward, to increase the $\mathrm{pH}$ of the solution up to 12 , Hydrazine Hydrate is gradually added and the color of the solution turned to dark brown.

After water evaporation by kept the solution in oven at $60{ }^{\circ} \mathrm{C}$ for $45 \mathrm{~min}$, the residual powder was washed with water and ethanol 3 times and again was kept in oven at $60{ }^{\circ} \mathrm{C}$ for $10 \mathrm{~h}$ until all the water was evaporated and the dark powder was left.

To easy distribution of $\mathrm{MoSe}_{2}$ powder in water, the obtained powder is stirred in alcohol for $12 \mathrm{~h}$, dried in the environmental conditions and finally, $5 \mathrm{mgr}$ of it dispersed in $10 \mathrm{cc}$ distilled water and sonicates to separate individual layers.

The transparent liquid above sediments that contain few/mono-layer of $\mathrm{MoSe}_{2}$ is achieved after centrifugation at $6000 \mathrm{rpm}$ for $3 \mathrm{~h}$. The quality of $\mathrm{MoSe}_{2}$ layers is investigated by TEM, SEM, EDX, Raman, FTIR and XRD analysis.

Figure 2a,b show the morphology of $\mathrm{MoSe}_{2}$ by SEM and TEM images. The few-layer structure of $\mathrm{MoSe}_{2}$ is seen in these pictures. Figure $2 \mathrm{c}$ implies the presence of Mo and Se in synthesized $\mathrm{MoSe}_{2}$ by EDX.

The Raman spectrum in Fig. 2d exhibits two peaks in $240\left(\mathrm{~cm}^{-1}\right)$ and $290\left(\mathrm{~cm}^{-1}\right)$ approximately that correspond to off-plane links $\left(\mathrm{A}_{1 \mathrm{~g}}\right)$ and intramolecular links $\left(\mathrm{E}_{2 \mathrm{~g}}\right)$ respectively ${ }^{31,34}$.

Figure 2e shows the graph of FTIR spectroscopy. In this figure $813\left(\mathrm{~cm}^{-1}\right), 992\left(\mathrm{~cm}^{-1}\right)$ and $1137\left(\mathrm{~cm}^{-1}\right)$ valleys are related to $\mathrm{Mo}-\mathrm{O}, \mathrm{Mo}=\mathrm{O}$ and $\mathrm{Se}-\mathrm{O}$ bonding respectively ${ }^{43}$.

Figure $2 \mathrm{f}$ illustrates the XRD spectrum peaks at $2 \theta=13.3^{\circ}, 25.82^{\circ}, 39.1^{\circ}$, which can be assigned to the (002), (004), and (103) planes of the hexagonal phase of $\mathrm{MoSe}_{2}$, respectively ${ }^{44,45}$.

$\mathrm{MoS}_{2}$. Two-dimensional $\mathrm{MoS}_{2}$ sheets synthesis by chemical exfoliation method. In this method, $\mathrm{MoS}_{2}$ was dispersed in a mixture of ethanol and water with $45 \%$ and $55 \%$ volume ratio. After stirring the composition for $10 \mathrm{~min}$, it was sonicated for $12 \mathrm{~h}$ in $40 \mathrm{~Hz}$ frequency to separate $\mathrm{MoS}_{2}$ sheets from each other. The quality of $\mathrm{MoS}_{2}$ layers is investigated by TEM, SEM, EDX, Raman, FTIR, and XRD analysis (Fig. 3). 

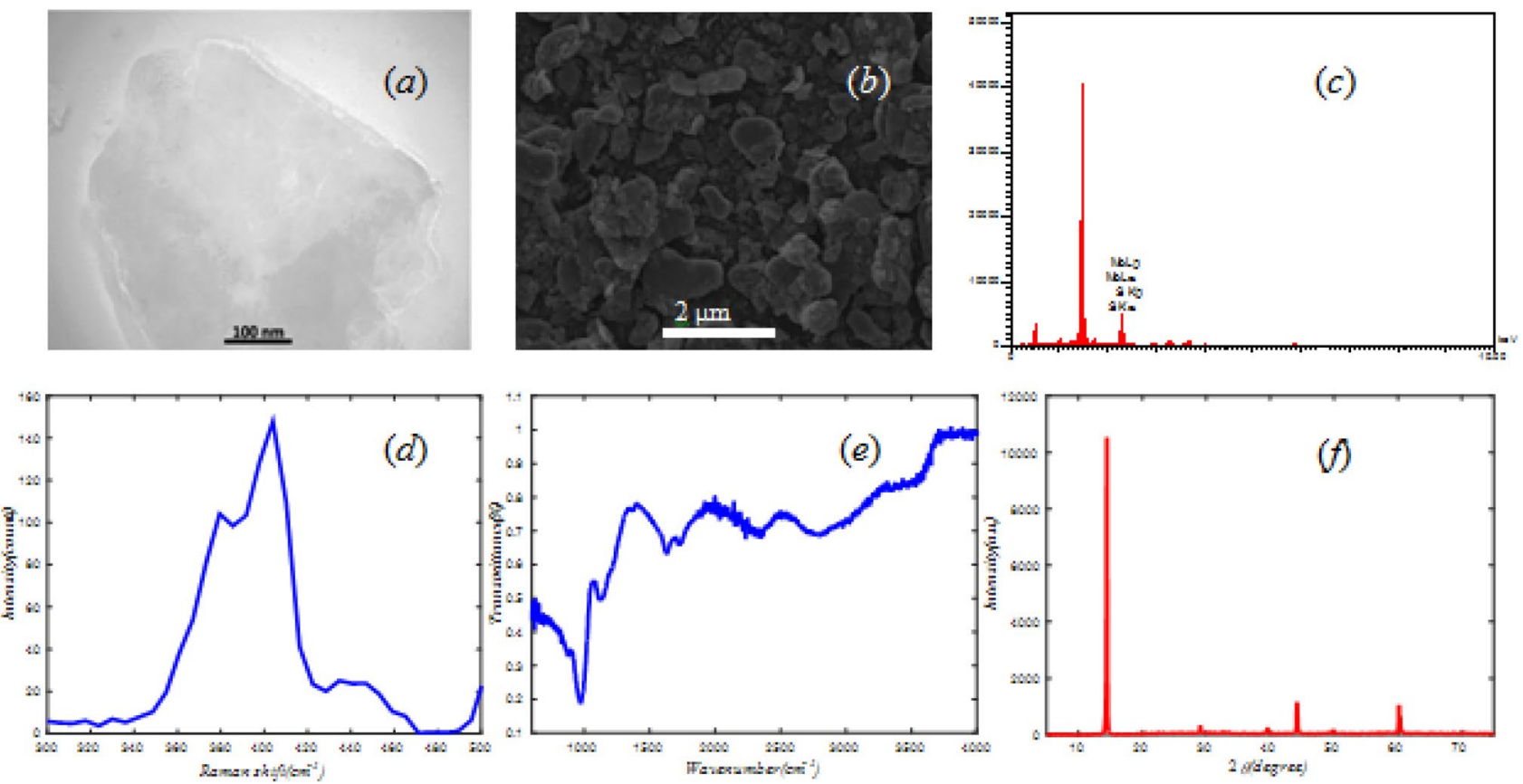

Figure 3. (a) TEM, (b) SEM, (c) EDX, (d) Raman, (e) FTIR, and (f) XRD analysis of $\mathrm{MoS}_{2}$.

The SEM and TEM images show the microstructure of synthesized $\mathrm{MoS}_{2}$ (Fig. 3a and Fig. 3b) and indicate to broad sheets of $\mathrm{MoS}_{2}$. The purity of $\mathrm{MoS}_{2}$ sheets is shown in the EDX spectrum (Fig. 3c). The Raman spectra of $\mathrm{MoS}_{2}$ layer (Fig. 3d) exhibit two important peaks, the in-plane $\mathrm{E}_{2 \mathrm{~g}}\left(\sim 381 \mathrm{~cm}^{-1}\right)$, and the out-of-plane $\mathrm{A}_{1 \mathrm{~g}}$ $\left(\sim 405 \mathrm{~cm}^{-1}\right) . \mathrm{E}_{2 \mathrm{~g}}$ mode corresponds to the Mo and $\mathrm{S}$ atoms vibrating in one direction, while $\mathrm{A}_{1 \mathrm{~g}}$ mode is due to the Sulfur atoms vibrating ${ }^{14,25}$. The difference between these two modes $\left(\sim 24 \mathrm{~cm}^{-1}\right)$ can be used as a reliable identification for few-layers $\mathrm{MoS}_{2}{ }^{46}$.

The FTIR spectrum (Fig. 3e) shows the presence of Mo-S, C-H, and Mo-O bands at 600, 1396, and $1639 \mathrm{~cm}^{-1}$ respectively. The appearance of a spread peak in the region of 3287 is related to the tensile vibration of $\mathrm{OH}$ groups $^{47}$.

The XRD of $\mathrm{MoS}_{2}$ sheets in Fig. 3f illustrates the peaks at $2 \theta=14^{\circ}(002), 2 \theta=32.6^{\circ}(100), 2 \theta=39.5^{\circ}(103)$ and $2 \theta=44.2^{\circ}(006), 2 \theta=49.8^{\circ}(105), 2 \theta=58.3^{\circ}(110)$. The peaks related to the $(002)$ plans determine the stacking of $\mathrm{MoS}_{2}$ single layer by d-spacing of $6.3 \mathrm{~nm}$. Other peaks are characteristic of crystalline nature of $\mathrm{MoS}_{2}$ materials ${ }^{47,48}$.

Fabrication of humidity sensor. In this research, SMF is used as the main part of the optical-humidity sensor. To modify the sensing, the diameter of SMF reduces from $34.45 \mu \mathrm{m}$ by corrosion. For this purpose, $3 \mathrm{~cm}$ of the protective layer of SMF was stripped and wiped up with alcohol, then put it in HF acid for 60 min. The process etched some of the clad-layer on the core of SMF to achieve the desirable evanescence field. Figure $4 a-c$ shows the SEM images of ESMF.

In order to make more changes in the refractive index by water absorption, 2D materials have deposited on the surface of ESMF by dip coating in transparent solution contain one or few layers of G/GO, $\mathrm{MoS}_{2}$ and $\mathrm{MoSe}_{2}$. These solutions are prepared by adding $5 \mathrm{mg}$ of synthesized powders to $10 \mathrm{cc}$ Distilled water separately, sonicating for $2 \mathrm{~h}$, and centrifuging. The SEM images of ESMF coated with G/GO, $\mathrm{MoSe}_{2}$ and $\mathrm{MoS}_{2}$ illustrate in Fig. $4 \mathrm{~d}-\mathrm{f}$ respectively. Also, the preparation of optical fiber for humidity sensing schematically is shown in Fig. 5.

The OPS and OPM are used as OLTS to analyze RDA, repeatability, sensitivity, and variance versus RH. To create the $\mathrm{RH}$ in the range of $20-90 \%$ in $25^{\circ} \mathrm{C}$, we used a cloud chamber consist of humidifier, humidity control box, temperature controller, and standard humidity sensor as shown in Fig. 5b. Due to the presence of temperature and humidity control system in cloud chamber, these quantities are uniform and constant throughout chamber.

\section{Result}

RDAs as a function of RH for ESMF with different coatings are illustrated in Fig. 6a and b for $1310 \mathrm{~nm}$ and $1550 \mathrm{~nm}$ wavelengths. As shown in these figures, ESMF coated with $\mathrm{MoS}_{2}$ has zero RDA at RH below $30 \%$ and at higher RH has lower RDA relative to ESMFs coated with $\mathrm{MoSe}_{2}$ and G/GO. So it cannot use as an efficient humidity sensor. Unlike it, the ESMF coated with $\mathrm{MoSe}_{2}$ has very good humidity sensing at low RH up to $30 \%$. However, the humidity sensor based on $\mathrm{MoSe}_{2}$ is not applicable at medium and high RH due to the lake of the one-to-one function of RH. The ESMF coated with G/GO has high RDA, especially at RH higher than $40 \%$, and also its RDA has the one-to-one function of RH. 

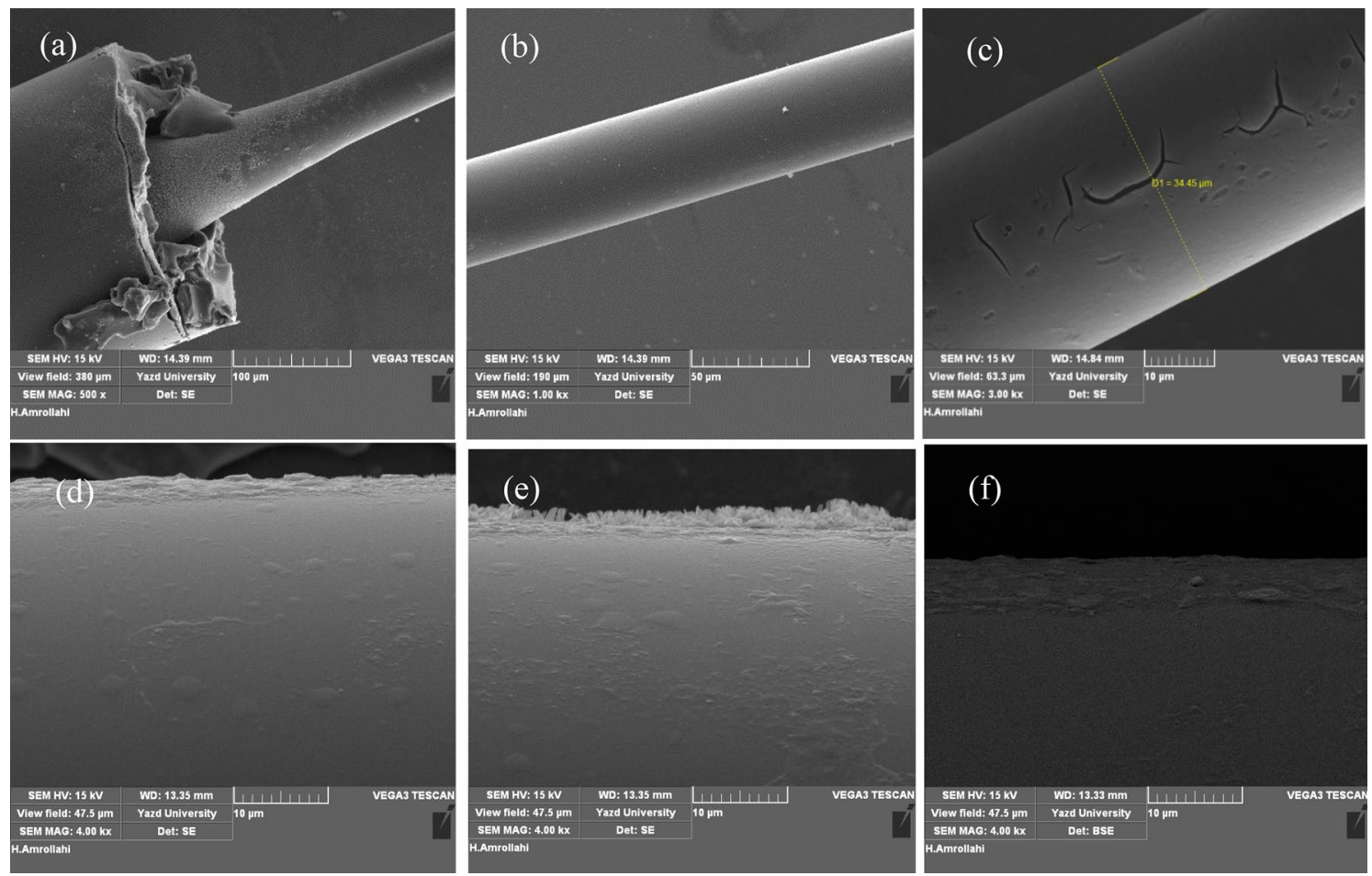

Figure 4. The SEM images of ESMF (a-c) and G/GO coated ESMF (d), MoSe ${ }_{2}$ coated ESMF (e), MoS coated $^{2}$ $\operatorname{ESMF}(\mathbf{f})$.

(a)

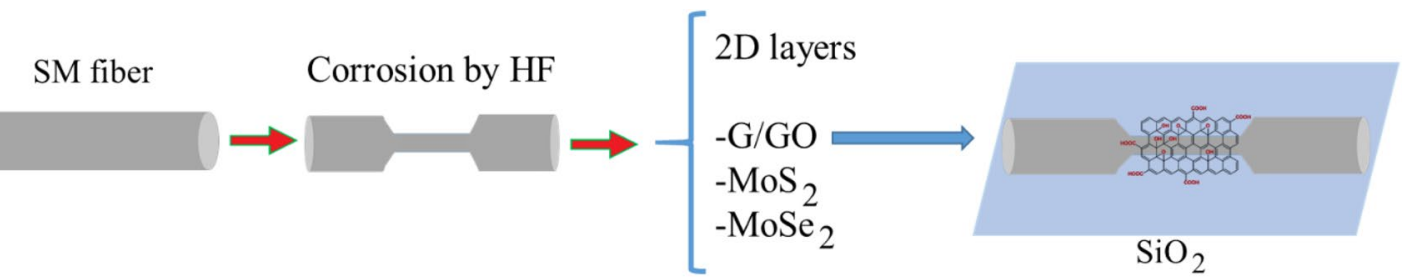

(b)

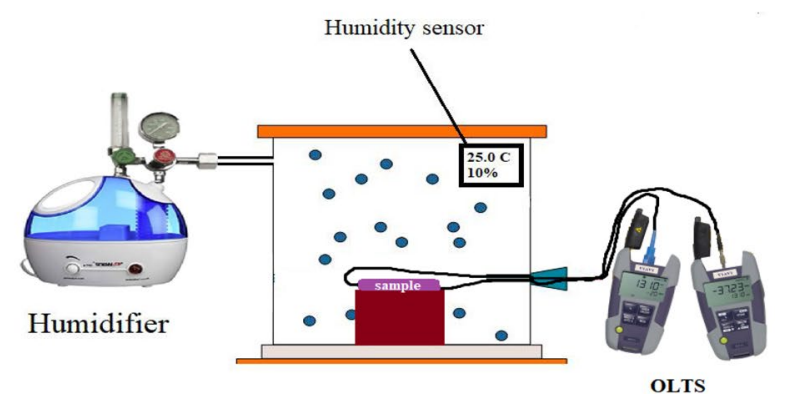

Figure 5. Schematic preparation of (a) optical fiber for humidity sensing and (b) the setup of the humidity sensing experiment.

The optical humidity sensing mechanism of coated ESMF is related to variation of refractive index by bonding between water molecules and the surface of $2 \mathrm{D}$ materials. This physical interaction changes the density of carriers in 2D materials coated on the ESMF and results in the variation of electronic gap energy $\left(\mathrm{E}_{\mathrm{g}}\right)$. According to the Penn model, $\mathrm{E}_{\mathrm{g}}$ are inversely correlated to refractive index ${ }^{49}$. 

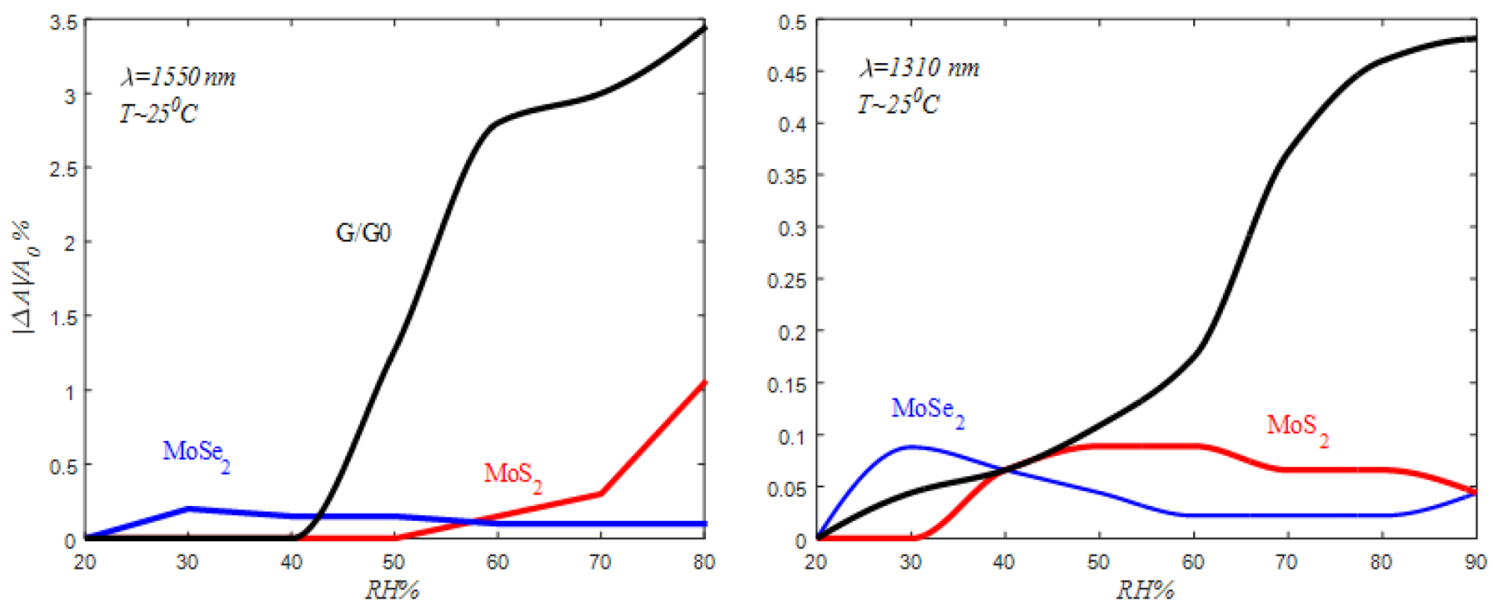

(a)

(b)

Figure 6. (a,b) RDA as a function of RH at room temperature with 1550 and $1310 \mathrm{~nm}$ wavelength for different coating respectively.

The presence of functional groups and defects determine the type of synthesized semiconductor powder. For example, impurities such as oxygen, water, carbon dioxide, etc. cause to p-type, and others such as hydroxyl, ether, and epoxide groups convert the intrinsic semiconductor to n-type. So, with respect to the FTIR spectrum (Fig. 1e), G/GO has hydroxyl groups and behaves as an n-type semiconductor. On the other hand, $\mathrm{MoSe}_{2}$ has not hydroxyl group and the presence of Mo bonds (Fig. 2e) at the surface makes it a p-type semiconductor. The existence of hydroxyl group and Mo bonds on the surface of $\mathrm{MoS}_{2}$ results in the simultaneous presence of acceptor and donor impurities.

The ionization of water molecules occurs as $2 \mathrm{H}_{2} \mathrm{O} \Leftrightarrow \mathrm{H}_{3} \mathrm{O}^{+}+\mathrm{OH}^{-}$near the surface by polar bonds. The negative part produces the water molecules by interacting with the Hydrogen in the air $\left(2 \mathrm{OH}^{-}+\mathrm{H}_{2} \rightarrow 2 \mathrm{H}_{2} \mathrm{O}\right)$. The positive part of it interacts with the hydroxyl group on the surfaces and excites as water molecules $\left(\mathrm{H}_{3} \mathrm{O}^{+}+\mathrm{OH}^{-} \rightarrow 2 \mathrm{H}_{2} \mathrm{O}\right)$. So, the adsorption of water molecules on the surface changes the carrier densities of it by withdrawing electrons.

Taking out the electrons from a p-type surface like $\mathrm{MoSe}_{2}$, by water adsorption, decreases the refractive index because of the increase in $\mathrm{E}_{\mathrm{g}}$. This mechanism in $\mathrm{G} / \mathrm{GO}$ as an n-type semiconductor is vice versa. However, $\mathrm{MoSe}_{2}$ coated ESMF at low humidity adsorb $\mathrm{H}_{2} \mathrm{O}$ and show high RDA relatively, and saturate in $\mathrm{RH}$ about $30 \%$. At higher humidity, $\mathrm{MoSe}_{2}$ saturate and will not adsorb more $\mathrm{H}_{2} \mathrm{O}$. As regards, the functional groups at G/GO can absorb $\mathrm{H}_{2} \mathrm{O}$ up to $90 \% \mathrm{RH}$ and the its refractive index increase by adsorption of water molecules on G/GO coated ESMF because of the increment of carrier densities.

The $\mathrm{MoS}_{2}$ coated ESMF has low RDA and indeterminate humidity sensing behavior because of competition between donor and acceptor of electrons impurities on its surface.

As shown in Fig. 6, RDA of G/GO in the telecommunication's standard wavelengths, $1310 \mathrm{~nm}$ and $1550 \mathrm{~nm}$, is higher than $\mathrm{MoSe}_{2}$, and $\mathrm{MoS}_{2}$. In addition to the changes in the density of carriers with respect to changes in relative humidity, the adsorption of water molecules between the graphene oxide sheets cause to change in refractive index and improve its humidity sensing ${ }^{50}$. So other quantities just measure for G/GO-based ESMF.

The repeatability curve shows a reduction of RDA during consecutive tests (Fig. 7a,c), due to saturation hydrogen bonding in G/GO structures. It seems thermal treatment of G/GO coated ESMF at $90{ }^{\circ} \mathrm{C}$ can reactive the sensor because of the separate the oxygen and water molecules from the G/GO surface ${ }^{16,51}$. To calculate the formulation for measuring RH from Attenuation, reversing curves are illustrated and fitted by MATLAB, which are shown in Fig. 7b,d.

Sensitivity has defined as a ratio between the differential of the output signal and measured properties (humidity) ${ }^{52}$. Sensitivity for the humidity sensors coated with G/GO, $\mathrm{MoSe}_{2}$ and $\mathrm{MoS}_{2}$ are illustrated in Fig. 8. It is obvious from this figure, sensitivity of G/GO $\left(\mathrm{MoS}_{2}\right)$ coted ESMF at RH more than $30 \%(60 \%)$ increases and has more magnitude in $1550 \mathrm{~nm}$ wavelength relative to $1330 \mathrm{~nm}$ wavelength. While, $\mathrm{MoSe}_{2}$ coated ESMF has very low sensitivity at both wavelengths.

Variances of any test from the average value are plotted in Fig. 9. As shown, variance for the investigated sensor is small in the RHs less than 40\%. In addition, variance is so little in $1310 \mathrm{~nm}$ wavelength than $1550 \mathrm{~nm}$. So, results obtained from the humidity sensor based on etched-fiber coated with G/GO have better accuracy at low humidity and $1310 \mathrm{~nm}$ wavelength. 

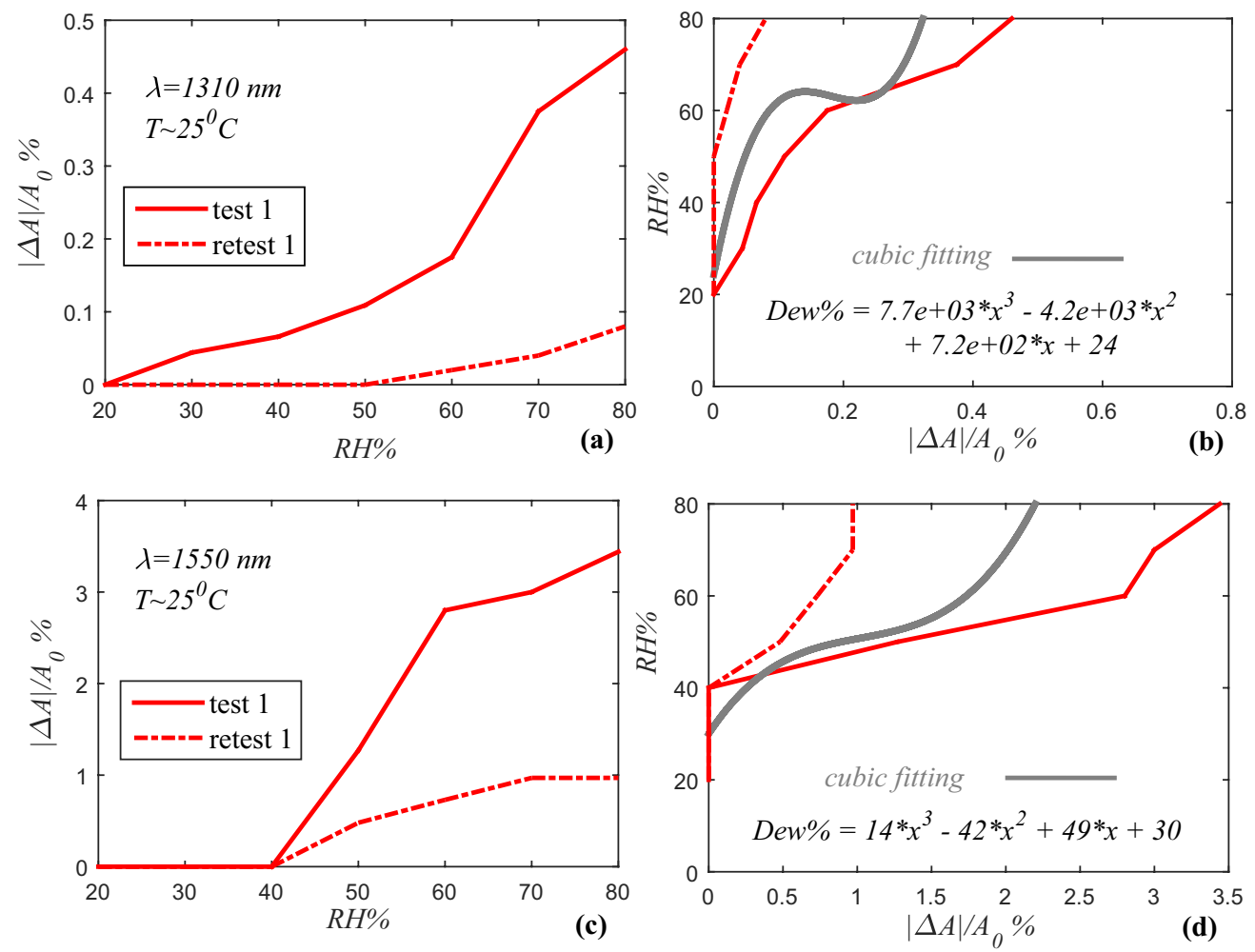

Figure 7. Repeatability of RDA as a function of RH for G/GO in $1310 \mathrm{~nm}$ (a) and $1550 \mathrm{~nm}$ (c) wavelengths; reversing and fitting curves to calculate RH formulation in $1310 \mathrm{~nm}(\mathbf{b})$ and $1550 \mathrm{~nm}$ (d) (dash-line curves are rapid retest of line curves).
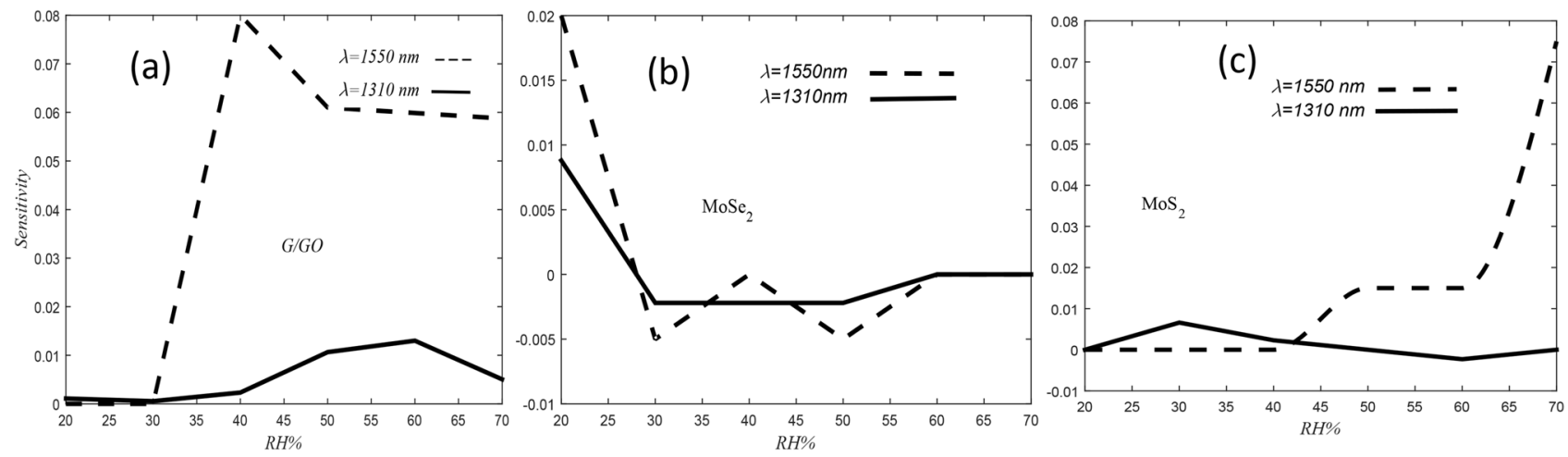

Figure 8. Sensitivity of humidity sensors coated with (a) G/GO, (b) $\mathrm{MoSe}_{2}$ and (c) $\mathrm{MoS}_{2}$ in both $1310 \mathrm{~nm}$ (line curves) and $1550 \mathrm{~nm}$ (dash-line curves) wavelengths.

\section{Conclusions}

In this research, ESMF that coated by G/GO, $\mathrm{MoSe}_{2}$, and $\mathrm{MoS}_{2}$ to application as humidity sensing are prepared. The RDA, repeatability, sensitivity, and variance analyzes are investigated versus RH at both standards wavelengths of telecommunication $(1310 \mathrm{~nm}$ and $1550 \mathrm{~nm})$. Results show that the sample coated with $\mathrm{MoSe}_{2} \mathrm{has}$ higher RDA at low humidity (less than $30 \%$ ). So it can be used as a low humidity sensor. However, the sensor based on $\mathrm{MoSe}_{2}$ is not practical at humidity more than $30 \%$ due to low and lake of the one-to-one function of RDA versus RH. The ESMF coated by $\mathrm{MoS}_{2}$ has indistinctive behavior by variation of RH because of $\mathrm{n}$-type and p-type impurities on the surface of $\mathrm{MoS}_{2}$. The best humidity sensor, in this research, is the ESMF that coated by G/GO. It has partially high and one by one function RDA versus RH. The sensitivity for it is reasonable and the low variance shows its accuracy. Also, repeatability can modify by thermal treatment. 


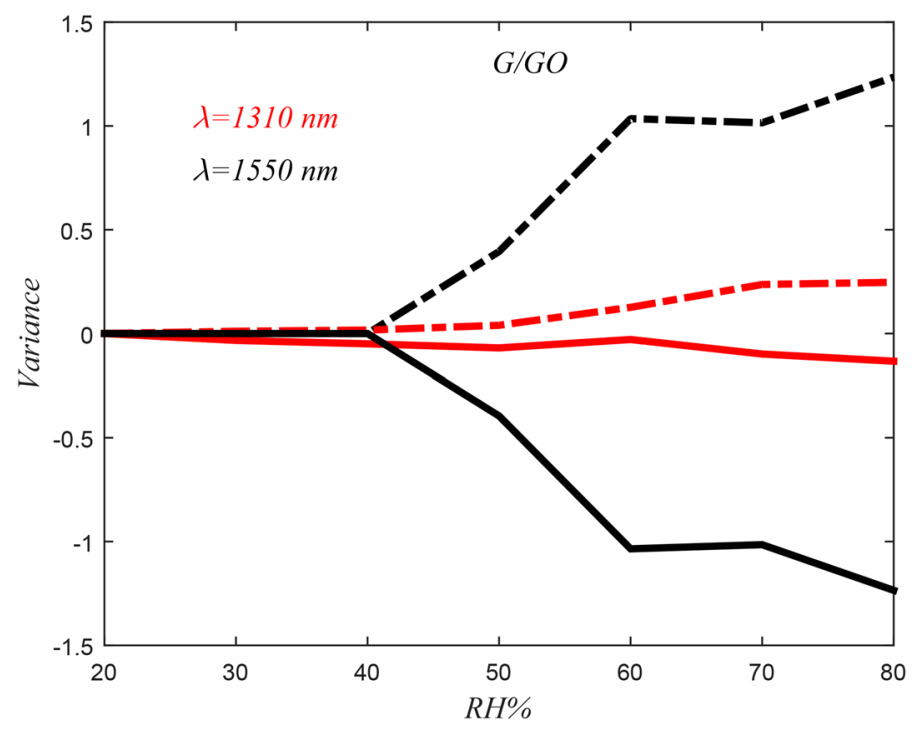

Figure 9. Variance as a function of RH from average values of G/GO-based sensor in $1310 \mathrm{~nm}$ and $1550 \mathrm{~nm}$ wavelengths.

Received: 16 July 2020; Accepted: 8 December 2020

Published online: 19 January 2021

\section{References}

1. Santos, J. L., Farahi, F. Hand Book of Optical Sensors 1st edn. 257-587 (CRC Press, 2018)

2. Cusano, A., Consales, M., Crescitelli, A., Ricciardi, A., (Eds.) Lab-on-Fiber Technology (Springer Series in Surface Sciences (56)) 27-40 (Springer, 2015)

3. Pisco, M. et al. Opto-mechanical lab-on-fibre seismic sensors detected the Norcia earthquake. Sci. Rep. 8, 6680 (2018).

4. Woyessa, G., Fasano, A., Markos, C., Rasmussen, H. K. \& Bang, O. Low loss polycarbonate polymer optical fiber for high temperature FBG humidity sensing. IEEE Photon. Technol. Lett. 29, 575-578 (2017).

5. Woyessa, G., Nielsen, K., Stefani, A., Markos, C. \& Bang, O. Temperature insensitive hysteresis free highly sensitive polymer optical fiber Bragg grating humidity sensor. Opt. Express. 24, 1206-1213 (2016).

6. Alberto, N. et al. Relative humidity sensing using micro-cavities produced by the catastrophic fuse effect. Opt. Quant. Electron. 48, 216 (2016).

7. Lopez-Torres, D. et al. Photonic crystal fiber interferometer coated with a PAH/PAA nanolayer as humidity sensor. Sens. Actuators B Chem. 242, 1065-1072 (2017).

8. Urrutia, A. et al. Simultaneous measurement of humidity and temperature based on a partially coated optical fiber long period grating. Sens. Actuators B Chem. 227, 135-141 (2016).

9. Ascorbe, J., Corres, J. M., Matias, I. R. \& Arregui, F. J. High sensitivity humidity sensor based on cladding-etched optical fiber and lossy mode resonances. Sens. Actuators B Chem. 233, 7-16 (2016).

10. Wei, W. et al. Graphene-based long-period fiber grating surface plasmon resonance sensor for high-sensitivity gas sensing. Sensors. 17, 2 (2017).

11. Huang, Y. et al. High-performance fibre-optic humidity sensor based on a side-polished fibre wavelength selectively coupled with graphene oxide film. Sens. Actuators B Chem. 255, 57-69 (2018).

12. Jiang, S. et al. A novel U-bent plastic optical fibre local surface plasmon resonance sensor based on a graphene and silver nanoparticle hybrid structure. J. Phys. D Appl. Phys. 50, 165105 (2017).

13. Saini, T. S. et al. Calcogenide W-type co-axial optical fiber for broadband highly coherent mid IR supercontiniuum generation. J. Appl. Phys. 124, 213101 (2018).

14. Wang, J. \& Wang, Q. Investigation electrical properties of controllable graphene nanoribbon field effect transistor. Phys. B Condens. Matter. 583, 412022 (2020)

15. Wei, L., Jun-fang, C., Qinyu, H. \& Teng, W. Electronic and elastic properties of $\mathrm{MoS}_{2}$. Phys. B Condens. Matter. 405, 2498-2502 (2010).

16. Hernaez, M. et al. Optical fibre sensors using graphene-based materials: A review. Sensors. 17, 155 (2017).

17. Novoselov, K. S. et al. Electric field effect in atomically thin carbon films. Science 306, 666-669 (2004).

18. Brumfiel, G. Graphene gets ready for the big time: Physicists are talking about how to make practical use of a former laboratory curiosity. Nature 458, 390-392 (2009).

19. Tatarova, E. et al. Microwave plasmas applied for the synthesis of free standing graphene sheets. J. Phys. D Appl. Phys. 47, 385501 (2014).

20. Suzuki, S. \& Yoshimura, M. Chemical stability of graphene coated silver substrates for surface-enhanced Raman scattering. Sci. Rep. 7, 14851 (2017).

21. Novoselov, K. S. et al. Two-dimensional gas of massless Dirac fermions in graphene. Nature 438, 197-200 (2005).

22. Ostovari, F., Abdi, Y., Darbari, S. \& Ghasemi, F. Effects of electromechanical resonance on photocatalytic reduction of the freehanging graphene oxide sheets. J. Nanopart. Res. 15, 1551 (2013). 
23. Ostovari, F. \& Moravvej-Farshi, M. K. Photodetectors with zigzag and armchair graphene nanoribbon channels and asymmetric source and drain contacts: detectors for visible and solar blind applications. J. Appl. Phys. 120, 144505 (2016).

24. Nag, A., Mitra, A. \& Mukhopadhyay, S. C. Graphene and its sensor-based applications: A review. Sens. Actuator A Phys. 270, 177-194 (2018).

25. Pei, S. \& Cheng, H. M. The reduction of graphene oxide. Carbon 50, 3210-3228 (2012).

26. Xiao, Y. et al. Reduced graphene oxide for fiber-optic humidity sensing. Opt. Express. 22, 31555-31567 (2014).

27. Gao, R. et al. Humidity sensor based on power leakage at resonance wavelengths of a hollow core fiber coated with reduced graphene oxide. Sens. Acuators B: Chem. 222, 618-624 (2016).

28. Huang, Y. et al. High-performance fibre-optic humidity sensor based on a side- polished fibre wavelength selectively coupled with graphene oxide film. Sens. Actuators B Chem. 255, 57-69 (2018).

29. Wang, Y. et al. Polarization-dependent humidity sensor based on an in-fiber Mach-Zehnder interferometer coated with graphene oxide. Sens. Acuators B: Chem. 234, 503-509 (2016).

30. Wang, Y. et al. Fiber optic relative humidity sensor based on the tilted fiber Bragg grating coated with graphene oxide. Appl. Phys. Lett. 109, 031107 (2016).

31. Shelke, N. T. \& Late, D. J. Hydrothermal growth of $\mathrm{MoSe}_{2}$ nanoflowers for photo- and humidity sensor applications. Sens. Actuator A Phys. 295, 160-168 (2019).

32. $\mathrm{Hu}, \mathrm{H}$. et al. Recent advances in two-dimensional transition metal dichalcogenides for biological sensing. Biosens. Bioelectron. 142, 111573 (2019).

33. Li, D. et al. Molybdenum disulfide nanosheets deposited on polished optical fiber for humidity sensing and human breath monitoring. Opt. Express. 25, 28407-24816 (2017).

34. Lang, Y., et al., Side polished fiber coated with molybdenum diselenide $\left(\mathrm{MoSe}_{2}\right)$ for humidity sensing. Advanced Photonics, SeW1E.2; https://doi.org/10.1364/SENSORS.2017.SeW1E.2 (2017).

35. Luo, Y. et al. Tungsten disulfide (WS $)$ based all-fiber-optic humidity sensor. Opt. Express. 24, 8956-8966 (2016).

36. Hummers, W. S. Jr. \& Offeman, R. E. Preparation of graphitic oxide. J. Am. Chem. Soc. 80, 1339 (1958).

37. Ostovari, F., Abdi, Y. \& Ghasemi, F. Controllable formation of graphene and graphene oxide sheets using photo-catalytic reduction and oxygen plasma treatment. EPJ Appl. Phys. 60, 30401 (2012).

38. Nossol, E. et al. Synthesis, characterization and morphology of reduced graphene oxide-metal-TCNQ nanocomposites. J. Mater. Chem. C. 2, 870-878 (2014).

39. Moon, I. K., Lee, J., Ruoff, R. S. \& Lee, H. Reduced graphene oxide by chemical graphitization. Nat. Commun. 1, 73 (2010),

40. Tito, H. A. R. et al. Electrical and optical properties of reduced graphene oxide thin film deposited onto polyethylene terephthalate by spin coating technique. Appl. Opt. 56, 7774-7780 (2017).

41. Gholampour, A., Valizadeh Kiamahalleh, M., Tran, D. N., Ozbakkaloglu, T. \& Losic, D. From graphene oxide to reduced graphene oxide: Impact on the physiochemical and mechanical properties of graphene-cement composites. ACS Appl. Mater. Interfaces. 9, 43275-43286 (2017)

42. Todica, M., Stefan, T., Simon, S., Balasz, I. \& Daraban, L. UV-Vis and XRD investigation of graphite-doped poly(acrylic) acid membranes. Turkish J. Phys. 38, 261-267 (2014).

43. Siddiqui, I. et al. Hydrothermally synthesized micron sized, broom-shaped $\mathrm{MoSe}_{2}$ nanostructures for superior photocatalytic water purification. Mater. Res. Express. 5, 125020 (2018).

44. Tang, H., Dou, K., Kaun, C. C., Kuang, Q. \& Yang, S. MoSe 2 nanosheets and their graphene hybrids: Synthesis, characterization and hydrogen evolution reaction studies. J. Mater. Chem. 2, 360-364 (2014).

45. Zhan, J. H., Zhang, Z. D., Qian, X. F., Xie, C. W. Y. \& Qian, Y. T. Synthesis of $\mathrm{MoSe}_{2}$ nanocrystallites by a solvothermal conversion from $\mathrm{MoO}_{3}$. Mater. Res. Bull. 34, 497-501 (1999).

46. Li, H. et al. From bulk to monolayer $\mathrm{MoS}_{2}$ : Evolution of Raman scattering. Adv. Funct. Mater. 22, 1385-1390 (2012).

47. Yi, M. \& Zhang, C. The synthesis of two-dimensional $\mathrm{MoS}_{2}$ nanosheets with enhanced tribological properties as oil additives. RSC Adv. 8, 9564-9573 (2018).

48. Mahdavi, M., Kimiagar, S. \& Abrinaei, F. Preparation of few-layered wide bandgap $\mathrm{MoS}_{2}$ with nanometer lateral dimensions by applying laser irradiation. Crystals. 10, 164 (2020).

49. Pan, D., Wan, Q. \& Galli, G. The refractive index and electronic gap of water and ice increase with increasing pressure. Nat. Commun. 5, 3919 (2014)

50. Liu, R., Gong, T., Zhang, K. \& Lee, C. Graphene oxide papers with high water adsorption capacity for air dehumidification. Sci. Rep. 7, 9761 (2017).

51. Arghir, I., Delport, F., Spasic, D. \& Lammertyn, J. Smart design of fiber optic surfaces for improved plasmonic biosensing. Natl. Biotechnol. 32, 473-484 (2015).

52. Chen, G. Y., Ding, M., Newson, T. \& Brambilla, G. A review of microfiber and nanofiber based optical sensors. Open Opt. J. 7, 32-57 (2013).

\section{Acknowledgements}

This research was supported by Shahid Ghandi Corporation Complex for technical measurement tools.

\section{Author contributions}

E.O., F.O., B.D. and N.S. synthesis the nano materials and prepared the setup of expriments. F.O. and E.O. wrote the main manuscript and prepared figures. H.M. and F.O. analyzed data. All authors reviewed the manuscript.

\section{Competing interests}

The authors declare no competing interests.

\section{Additional information}

Correspondence and requests for materials should be addressed to H.M.

Reprints and permissions information is available at www.nature.com/reprints.

Publisher's note Springer Nature remains neutral with regard to jurisdictional claims in published maps and institutional affiliations. 
(c) (i) Open Access This article is licensed under a Creative Commons Attribution 4.0 International cc) License, which permits use, sharing, adaptation, distribution and reproduction in any medium or format, as long as you give appropriate credit to the original author(s) and the source, provide a link to the Creative Commons licence, and indicate if changes were made. The images or other third party material in this article are included in the article's Creative Commons licence, unless indicated otherwise in a credit line to the material. If material is not included in the article's Creative Commons licence and your intended use is not permitted by statutory regulation or exceeds the permitted use, you will need to obtain permission directly from the copyright holder. To view a copy of this licence, visit http://creativecommons.org/licenses/by/4.0/.

(C) The Author(s) 2021 